\title{
Highly Efficient Rinse-Off/Leave-On Scalp Care Treatments to Reduce Moderate to Severe Dandruff
}

\author{
Dorothea Schweiger ", Frank Rippke, Philip Drescher, Sandra Braren, Juliane Lüttke, \\ Alexander Filbry, Heiner Max
}

Research \& Development, Beiersdorf AG, Hamburg, Germany.

Email: *dorothea.schweiger@beiersdorf.com

Received November $23^{\text {rd }}, 2012$; revised December $25^{\text {th }}, 2012$; accepted January $5^{\text {th }}, 2013$

\begin{abstract}
Background/Aim: Dandruff is a common scalp problem associated with flaky and inflamed skin. In this study, we investigated the efficacy of a shampoo containing piroctone olamine and climbazole and the combination of this shampoo with a scalp tonic containing piroctone olamine and licochalcone A, derived from Glycyrrhiza inflata root extract, to reduce dandruff. Study Design/Methods: After conditioning, 102 subjects ( $\delta 56$ and 46 ( ) with moderate to very strong dandruff affection underwent a randomized half head study for 4 weeks. The volunteers' heads were washed regularly either with a placebo or the anti-dandruff shampoo, followed by the anti-dandruff tonic application or no treatment. In a 2-week post-treatment phase, subjects only applied placebo shampoo. Every two weeks, trained experts assessed dandruff affection based on a scale of 1 to 6 . At study start and after every 2 weeks, cytokine concentrations and anti-fungal activity of test products were determined in scalp wash-ups by enzyme-linked immunosorbant assays or a Malassezia colony-forming assay, respectively. Results: Results of expert grading and anti-fungal activity revealed a significant reduction in dandruff affection and Malassezia colony-forming units after treatment with the anti-dandruff shampoo or its combination with the tonic. Dandruff affection even remained reduced in the post-treatment phase when levels of Malassezia colony-forming units had returned to baseline. Cytokine analyses proved a significant decrease in pro-inflammatory dandruff markers after treatment with both anti-dandruff products. For the shampoo/tonic combination, a superior reduction of one scalp inflammatory marker was determined even after the post-treatment phase. Conclusion: Both the rinse-off shampoo as well as its combination with the leave-on tonic excellently alleviated dandruff and its associated micro-inflammation. Both treatments showed anti-fungal activity. The superior benefit, exerted by the combination, is primarily based on the known anti-inflammatory effect of licochalcone A and the improved scalp substantivity of the leave-on application.
\end{abstract}

Keywords: Dandruff; Rinse-Off Shampoo; Leave-On Tonic; Anti-Mycotics; Licochalcone A

\section{Introduction}

Dandruff represents a common scalp disorder affecting half of the world's population regardless of gender or ethnicity [1]. In contrast to normal healthy scalp, dandruff is characterized by patches of loosely adherent flakes and often accompanied by pruritus [1,2]. Patients suffering from dandruff are frequently diagnosed with seborrhoeic dermatitis [3]. Since dandruff is a chronic relapsing condition, this disorder has a considerable impact on individual quality of life [4].

The etiology of dandruff is dependent on the following factors: sebum secreted by sebaceous glands, metabolization of sebum by Malassezia species leading to the production of irritating unsaturated free fatty acids, and the individual susceptibility to these free fatty acids and

*Corresponding author. subsequent inflammation [5]. Dandruff symptoms are believed to be the result of a disturbed barrier function, which leads to (micro)-inflammation, and thus interferes with normal cell proliferation and differentiation [6].

The most widely utilized treatment options are antidandruff shampoos [7]. The suspected role of Malassezia species in the formation of dandruff has led to the incorporation of anti-mycotics in these special shampoos. Most of these anti-fungals inhibit the growth of Malassezia species. Actives include azoles, hydroxypyridones, coal tar, salicylic acid, sulfur, selenium sulfide, and zinc pyrithione [1].

In this context, it is conceivable that symptoms might be reduced even more effectively in case a topical leaveon formulation is applied after shampooing.

To effectively treat dandruff in the long term, an ideal leave-on formulation for the application after shampooing 
needs to 1) exert anti-fungal actions, 2) decrease microinflammation to help restore normal cell proliferation, and 3 ) reduce pruritus.

Taking these prerequisites into account, we created a leave-on tonic containing: 1) the soluble piroctone olamine, which belongs to the class of hydroxypyridone anti-mycotics, 2) the flavonoid licochalcone A to control focal (micro)-inflammatory processes, and 3) the local anesthetic polidocanol, which is well known to alleviate itch $[8,9]$. Licochalcone A represents a component isolated from the root of the licorice species Glycyrrhiza inflata. Regarding human skin, licochalcone A has been associated with anti-inflammatory effects in vitro $[10,11]$ and in vivo [11,12]. A recent study, performed in our laboratory, demonstrated the significant improvement of scalp condition after application of a leave-on tonic containing urea, lactate, polidocanol, and licochalcone A in the treatment of the dry, itchy, and subclinically inflamed scalp. The tonic increased skin hydration, decreased pruritus, normalized lipid changes, and reduced levels of pro-inflammatory markers [13].

Here, we determined the effects of the application of a picroctone olamine-, climbazole-, and polidocanol-containing shampoo followed by a treatment with the new leaveon tonic in subjects with moderate to severe dandruff. Results showed that the combination treatment with the antifungal shampoo and the new leave-on tonic reduced dandruff-associated scalp flaking and micro-inflammation even more effectively and over an extended period of time than shampooing alone.

\section{Materials and Methods}

\subsection{Test Products}

Three different formulations (2 shampoos and 1 tonic) were utilized in the course of this in vivo study. The verum shampoo (Eucerin ${ }^{\circledR}$-DermoCapillaire Anti-Dandruff Gel Shampoo) contained piroctone olamine, climbazole and polidocanol as active ingredients (INCI: aqua, sodium laureth sulfate, cocamidopropyl betaine, laureth-9, piroctone olamine, climbazole, undecylenamido propyl betaine, polyquaternium-10, glycerin, sodium chloride, citric acid, PEG-40 hydrogenated castor oil, PEG-200 hydrogenated glyceryl palmate, sodium benzoate, sodium salicylate, parfum). The placebo shampoo was of similar composition as the verum shampoo, but did not comprise any actives. This shampoo was also used for standardization purposes during the conditioning phase prior to the study start and during the post-treatment phase. The topical aqueous leave-on tonic (Eucerin ${ }^{\circledR}$-DermoCapillaire Anti-Dandruff Intensive Scalp Treatment) contained piroctone olamine, licochalcone A, derived from Glycyrrhiza inflata root extract, and polidocanol as active ingredients (INCI: aqua, alcohol denat. laureth-9, piroc- tone olamine, Glycyrrhiza inflata root extract, PEG-40 hydrogenated castor oil, hydroxyethylcellulose, citric acid).

\subsection{Determination of Shampoo and Tonic Effects in Vivo}

One hundred thirty-eight volunteers, known from earlier studies to normally suffer from dandruff, were recruited to participate in this study. After completion of a 14-day conditioning phase, 102 volunteers (56 male and 46 female, 21 - 65 years) displayed sufficient dandruff affection to be enrolled in the study (showing at least "moderate" dandruff). The aim of this study was to determine the amount of dandruff present on the scalp after application of the placebo shampoo, the anti-dandruff test shampoo alone, or the anti-dandruff test shampoo in combination with the leave-on anti-dandruff tonic. Trained experts performed visual assessment of the dandruff affection of the scalp surface based on the following assessment scale: $1=$ no dandruff; $2=$ single dandruff visible; $3=$ slight dandruff affection; $4=$ moderate dandruff affection; $5=$ strong dandruff affection; $6=$ very strong dandruff affection.

In this in vivo study, the recommendations of the current version of the Declaration of Helsinki and the guideline of the International Conference on Harmonization Good Clinical Practice (ICH GCP) were observed as applicable to a non-drug study. All volunteers provided written, informed consent. The study was conducted in a randomized, investigator-blinded, split head design and carried out under dermatological supervision. The split head design followed an incomplete block design.

To standardize scalp condition prior to the study start, all volunteers took part in a 14-day conditioning phase. In the course of the 4-week treatment phase, test formulations were applied by experienced hair dressers 3 times a week at the Institute Schrader test studio for applied hair cosmetics (Holzminden, Germany) according to written instructions. Test sites were the right and the left sides of the head. Approximately $0.5 \mathrm{~cm}$ of the middle head area was not treated to avoid cross-contamination of test formulations. Consequently, in this middle head area no measurements were performed or samples were taken.

Trained experts washed the volunteers' half heads with placebo or verum shampoo according to a randomization scheme. After $1 \mathrm{~min}$ application time, the two halves were separately and thoroughly rinsed with water. This procedure was followed by the randomized application of the leave-on tonic or no treatment. For the application of the tonic (if required), the hair was lifted at several positions with the help of a comb, and the tonic was directly spread on the scalp utilizing a syringe $(2$ to $5 \mathrm{ml})$. 
After that, the scalp was massaged, and the hair was dried with a hair dryer. Seventy-two hours prior to measurements, no tonic was applied. Assessment of dandruff affection (expert grading) was performed at 2-week intervals at baseline $\left(\mathrm{t}_{0}\right)$ and after $2\left(\mathrm{t}_{2}\right)$ and $4\left(\mathrm{t}_{4}\right)$ weeks of treatment.

After having completed 4 weeks of treatment, volunteers entered the post-treatment phase. During this period, volunteers washed their hair 3 times a week at home using the placebo shampoo without any actives. After 2 weeks $\left(\mathrm{t}_{6}\right)$, the individual dandruff affection on both halves of the head was evaluated by the trained experts again.

\subsection{Determination of Scalp Cytokine Levels}

At study start $\left(\mathrm{t}_{0}\right), 37$ out of 102 volunteers ( 25 male, 12 female; $23-65$ years) showed strong or very strong dandruff affection on one or both of their scalp sides. These volunteers were selected for additional cytokine analyses.

For the investigation of interleukin $1 \alpha$ (IL-1 $\alpha)$, interleukin 1 receptor antagonist (IL-1ra), and interleukin 8 (IL-8) levels, two aqueous standardized scalp wash-up samples were taken from volunteers' half scalp sides at $t_{0}$, $\mathrm{t}_{2}, \mathrm{t}_{4}$ and $\mathrm{t}_{6}$.

The scalp wash-up samples were processed as previously described [13]. Briefly, the hair of each volunteer was parted in the test area and secured. The scalp was gently and repeatedly rubbed with a standard cotton bud moistened with a rinse-off buffer. Shortened cotton buds were placed into shortened pipette tips and the remaining liquid in the buds collected by centrifugation. Corresponding wash-up samples were combined. For analysis, samples obtained from the same test sites of the same volunteer at the same point in time were pooled as well.

Total protein content was analyzed using the Pierce ${ }^{\circledR}$ BCA Protein Assay Kit (Thermo Scientific, Rochester, NY, USA) according to the manufacturer's instructions. For the determination of IL- $1 \alpha$ and IL-1ra levels, commercially available sandwich ELISAs (enzyme-linked immunosorbent assay; Quantikine ${ }^{\circledR}$ Human Immunoassay; R\&D Systems, Wiesbaden, Germany) were utilized according to the manufacturer's protocol. Results were normalized to the individual total protein content of the respective sample. For analysis of IL-8 levels, the ELISA (enzyme-linked immunosorbent assay) Opt EIA ${ }^{\mathrm{TM}}$ Human IL-8 (BD Bioscience, Heidelberg, Germany) was used. The coating buffer was slightly modified and consisted of $4.5 \mathrm{~g} \mathrm{NaHCO}_{3}$ and $1.78 \mathrm{~g} \mathrm{Na}_{2} \mathrm{CO}_{3}$ (both obtained from Merck, Darmstadt, Germany) ad 0.51 demineralized $\mathrm{H}_{2} \mathrm{O}$. All other steps and procedures were carried out as recommended by the manufacturer.

\subsection{Determination of Anti-Fungal Activity of Test Products}

To analyze anti-fungal activity of the test products, one standardized scalp wash-up was collected from every half scalp side of each volunteer at $\mathrm{t}_{0}, \mathrm{t}_{2}, \mathrm{t}_{4}$, and $\mathrm{t}_{6}$.

In brief, samples were obtained using a sterile swab system (Copan Diagnostics Inc., Murrieta, CA, USA) filled with $3 \mathrm{ml}$ rinse-off buffer $\left(12.49 \mathrm{~g} / 1 \mathrm{Na}_{2} \mathrm{HPO}_{4}, 0.63\right.$ $\mathrm{g} / 1 \mathrm{KH}_{2} \mathrm{PO}_{4}, 1 \mathrm{~g} / 1$ Triton X-100; all obtained from Sigma, Steinheim, Germany). For sampling, the hair was parted with the help of a tail comb. Immediately, the swab tip, which was moistened with rinse-off buffer, was run 10 times across the scalp covering an area of approx. 2 - 4 $\mathrm{cm}$. After sample collection, each swab was placed in the corresponding container and samples were vortexed. This procedure was repeated twice.

Using a spiral plater (Meintrup DWS Laborgeräte $\mathrm{GmbH}$, Lähden-Holte, Germany), $100 \mu \mathrm{l}$ of sample-containing solution were transferred to agar plates made of $10 \mathrm{~g} / \mathrm{l}$ pepton (Thermo Scientific), $6 \mathrm{~g} / \mathrm{l}$ glucose, $5 \mathrm{~g} / \mathrm{l}$ ox bile, $0.1 \mathrm{~g} / 1$ yeast extract, $1 \mathrm{ml}$ glycerol, $0.5 \mathrm{~g} / 1$ glycerol monostearat, $0.5 \mathrm{~g} / 1$ Tween $60,12 \mathrm{~g} / 1$ agar (all obtained from Merck), $10 \mathrm{ml} 3.5 \%$ cow's milk (local supermarket), as well as $2.5 \mathrm{mg} / \mathrm{l}$ gentamicin, $100 \mathrm{mg} / \mathrm{l}$ chloramphenicol, $400 \mathrm{mg} / \mathrm{l}$ cycloheximide (all obtained from Sigma).

Plates were then incubated at $32^{\circ} \mathrm{C}$ for 14 days. Plates displaying a $\mathrm{CFU} / \mathrm{ml}$ lower than 100 colonies at study start were excluded from further analyses.

\subsection{Statistical Analysis}

A significance level of 0.05 (alpha) was chosen for statistical analysis, based on two-sided hypothesis testing. For analysis, SAS software package for Windows V9.2. and SigmaPlot 11.0 (Systat Software, Inc., Chicago, IL, USA) were used.

Due to the incomplete block design the data were assumed to be independent according to the products for the statistical analysis. This approach is conservative, so the statistical results can be regarded as valid. The data of the expert grading and Malassezia colony formation were analyzed by Wilcoxon's rank test. The cytokine data were checked for normal distribution by means of Shapiro-Wilk test. Due to the fact that the hypothesis of normality was rejected for the interleukin data, the Blomtransformed ranks of the original data were used for analyses. In order to suitably model intra-individual changes, treatment comparisons were performed by incorporating baseline values. The initial situation at baseline $t_{0}$ was evaluated by analysis of variance, using "treatment" as explanatory variable. The treatment effects were evaluated by analysis of (co)variance. The treated vs. placebo control comparison was performed by analysis of covariance with "treatment" and " $\mathrm{t}_{0}$ " as explanatory 
variables, where "treatment" was the classification variable and " $t_{0}$ " the covariate. The comparison with baseline was performed by repeated measure analysis of variance with "point in time" as classification variable. If applicable, post hoc pairwise comparisons were performed.

\section{Results}

\subsection{Determination of Shampoo and Tonic Effects in Vivo}

Volunteers showing moderate to very strong dandruff affection participated in this in vivo study, which was performed to determine the efficacy of placebo shampoo, verum shampoo alone or verum shampoo in combination with tonic treatment to treat dandruff. Each volunteer tested two different formulations on split head with a randomization following the incomplete block design, so that each formulation or the combination of verum shampoo and tonic was applied to 48 - 51 heads. Figure 1 illustrates, that on average the scalps at baseline $\left(t_{0}\right)$ showed strong dandruff affection in all groups. At base- line, all dandruff scores were comparable (placebo vs. verum shampoo: $\mathrm{t}_{0}: \mathrm{p}=0.8137$, placebo shampoo vs. verum shampoo and tonic: $\mathrm{t}_{0}: \mathrm{p}=0.2888$, verum shampoo vs. verum shampoo and tonic: $\mathrm{t}_{0}: \mathrm{p}=0.3872$ ).

All formulations reduced dandruff affection during the anti-dandruff treatment. However, after 4 weeks of treatment, expert grading regarding the amount of dandruff on scalp revealed that volunteers treated with the verum shampoo showed a significantly lower dandruff score than volunteers who had applied the placebo shampoo $\left(\mathrm{t}_{4}\right.$ : $\mathrm{p}=0.0033$ ). In comparison to placebo, volunteers treated with the verum shampoo in combination with the tonic displayed a significant decrease in the dandruff score already starting after 2 weeks of treatment $\left(t_{2}: p=0.0200\right.$, $\left.\mathrm{t}_{4}: \mathrm{p} \leq 0.0001\right)$. After completion of the post-treatment phase, this significant reduction shown for both antidandruff treatments in comparison to placebo was maintained (verum shampoo: $\mathrm{t}_{6}: \mathrm{p}=0.0119$, verum shampoo and tonic: $\left.\mathrm{t}_{6}: \mathrm{p}=0.0004\right)$. However, treatment with the verum shampoo alone did not reduce dandruff affection on scalp as effectively as the treatment combination.
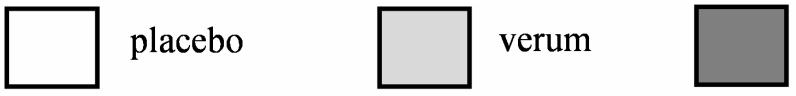

verum and tonic

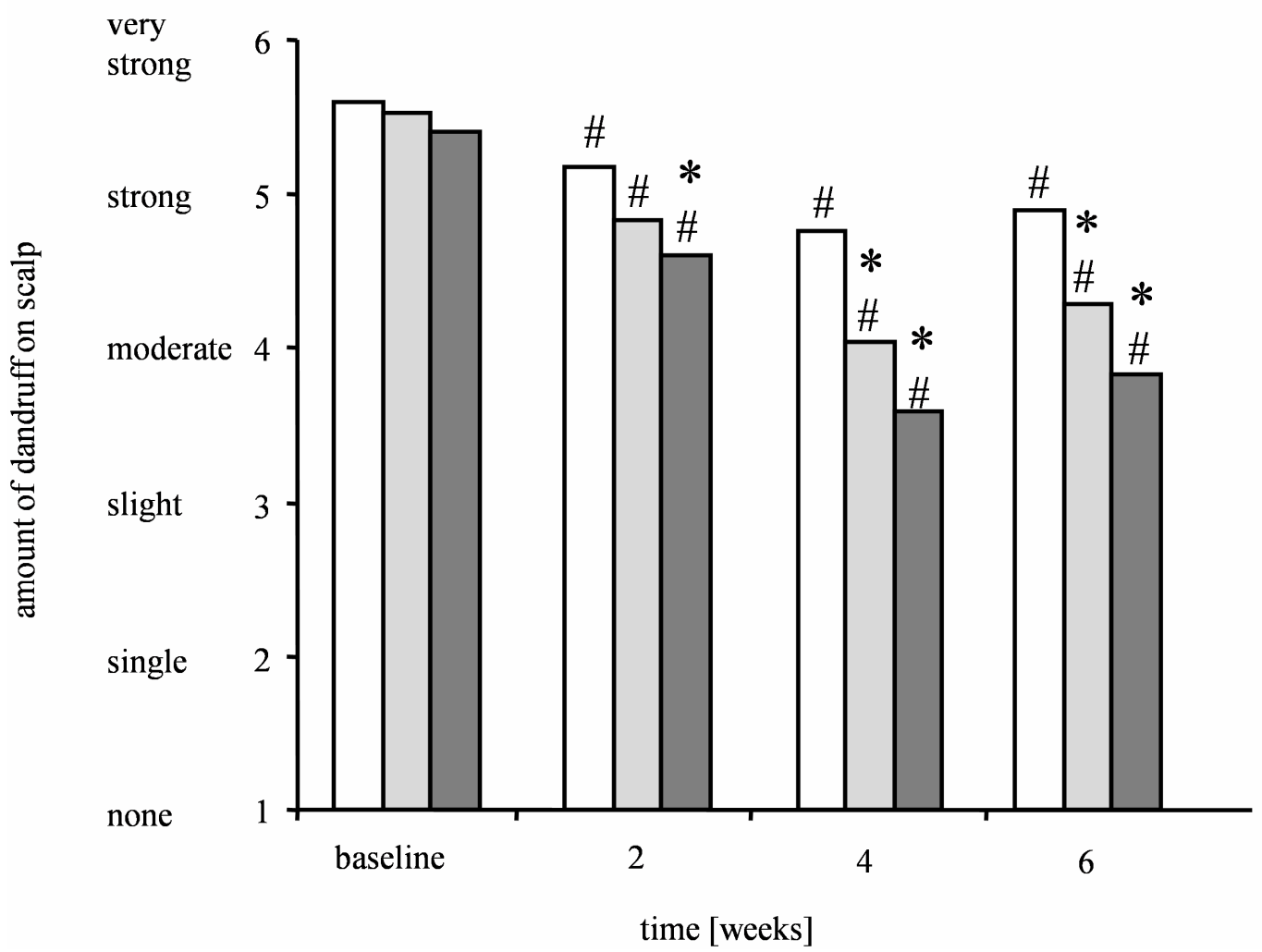

Figure 1. Decrease in the amount of dandruff after treatment with placebo, verum, or verum shampoo in combination with tonic as determined by expert grading. Results are shown as mean for each treatment group $(n=48-51)$. Significant differences with respect to placebo shampoo are marked with an asterisk (* for $p \leq 0.05)$ and with respect to baseline $t_{0}$ with a hash ( ${ }^{\#}$ for $\mathbf{p} \leq \mathbf{0 . 0 5}$ ). 
The dandruff score was significantly decreased compared to baseline $\left(\mathrm{t}_{0}\right)$ starting after 2 weeks of treatment with the verum shampoo and the verum shampoo in combination with the tonic $\left(\mathrm{t}_{2}-\mathrm{t}_{6}: \mathrm{p} \leq 0.0001\right)$. At these points in time, the placebo shampoo yielded a significant reduction in dandruff affection compared to baseline $\left(t_{0}\right)$ as well $\left(\mathrm{t}_{2}-\mathrm{t}_{6}: \mathrm{p} \leq 0.0091\right)$.

The moderate to strong dandruff affection was decreased to slight dandruff affection after the 4-week treatment with the verum shampoo or the verum shampoo/ tonic combination with the combination reaching almost single dandruff affection (grade 2). Application of placebo shampoo only induced a limited dandruff-reducing effect leading to a moderate dandruff score (grade 4).

The study results showed that test formulations were well tolerated by the study participants. Three volunteers dropped out of the study out of personal reasons and one volunteer discontinued the study due to incompatibility problems during the first 2 weeks after treatment with the combination of verum shampoo and tonic. These volunteers were not included into the study analysis.

\subsection{Determination of Scalp Cytokine Levels}

To determine effects of the test formulations on IL-1ra/ IL-1 $\alpha$ ratios and IL-8 levels, scalp wash-ups were taken from 37 volunteers with strong and very strong dandruff scores at $t_{0}, t_{2}, t_{4}$, and $t_{6}$. Due to the incomplete block design, samples were taken from 19 half head sites treated with the verum shampoo, 20 half heads, which received the placebo shampoo, and 17 half heads, which were treated with the verum shampoo in combination with the tonic. At baseline, all values were comparable (placebo vs. verum shampoo: $\mathrm{t}_{0}: \mathrm{p}=0.7429$, placebo shampoo vs. verum shampoo and tonic: $p=0.7642$, verum shampoo vs. verum shampoo and tonic: $p=0.5416$ ).

As shown in Figure 2(a), a significant reduction of the cytokine ratio IL-1ra/IL- $1 \alpha$ was achieved after 4 and 6 weeks of treatment with the verum shampoo as well as with the combination of verum shampoo and tonic in comparison to baseline (verum shampoo: $\mathrm{t}_{4}: \mathrm{p}=0.0001$, $\mathrm{t}_{6}: \mathrm{p}=0.0003$; verum shampoo and tonic: $\mathrm{t}_{4}: \mathrm{p}=0.0001$, $\left.\mathrm{t}_{6}: \mathrm{p} \leq 0.0001\right)$. Compared to placebo shampoo, the IL-1ra/IL- $1 \alpha$ ratio was significantly decreased after application of the verum shampoo at points in time $t_{2}(p=$ $0.0275), t_{4}(p=0.0010)$, and $t_{6}(p=0.0001)$. Regarding the treatment with verum shampoo and tonic, the IL-1ra/ IL-1 $\alpha$ ratio was significantly reduced compared to placebo after 4 and 6 weeks $\left(\mathrm{t}_{4}: \mathrm{p}=0.0157, \mathrm{t}_{6}: \mathrm{p}=0.0002\right)$.

The decrease in pro-inflammatory IL-8 levels after treatment with the test formulations is illustrated in Figure 2(b). The fact that data were significantly different at baseline (placebo vs. verum shampoo: $\mathrm{t}_{0}: \mathrm{p}=0.0147$, placebo shampoo vs. verum shampoo and tonic: $\mathrm{p}=$ 0.4006 , verum shampoo vs. verum shampoo and tonic: $p$ $=0.0020$ ) was taken into account by analysis of covariance with covariate $\mathrm{t}_{0}$.

A significant reduction of IL-8 levels compared to baseline was found after treatment with the verum shampoo, as well as the combination of verum shampoo and tonic at all points in time (verum shampoo: $\mathrm{t}_{2}: \mathrm{p}=0.0049, \mathrm{t}_{4}: \mathrm{p}$ $\leq 0.0001, \mathrm{t}_{6}: \mathrm{p}=0.0400$; verum shampoo and tonic: $\mathrm{t}_{2}, \mathrm{t}_{4}$ and $\left.t_{6}: p \leq 0.0001\right)$. Also, compared to placebo shampoo a significant decrease in IL-8 levels was determined after a 2 - and 4-week treatment with the verum shampoo $\left(t_{2}\right.$ and $\left.t_{4}: p \leq 0.0001\right)$. After the post-treatment phase $t_{6}$, however, this reduction was no longer significant $\left(t_{6}: p=\right.$ 0.0707). The application of the combination of verum shampoo and tonic resulted in a significant reduction in IL-8 levels at all points in time, even after completion of the post-treatment phase $\left(\mathrm{t}_{2}: \mathrm{p} \leq 0.0001, \mathrm{t}_{4}: \mathrm{p}=0.0026, \mathrm{t}_{6}\right.$ : $\mathrm{p}=0.0048)$.

\subsection{Determination of Anti-Fungal Activity}

For the detection of anti-fungal activity of different test products, a Malassezia colony-forming assay using scalp wash-ups was carried out. Due to the incomplete block design and a poor colony formation $(\mathrm{CFU} / \mathrm{ml}<100)$ in a few plates at study start, scalp samples obtained from 23 half head sites treated with placebo shampoo, 21 half heads treated with verum shampoo, and 24 half heads treated with the combination of verum shampoo and tonic were analyzed. Reductions in sample numbers during the study are due to a few drop outs of volunteers at specific points in time.

Starting at comparable baseline levels (placebo vs. verum shampoo: $\mathrm{p}=0.8786$; placebo shampoo vs. verum shampoo and tonic: $\mathrm{p}=0.9153$; verum shampoo vs. verum shampoo and tonic: $\mathrm{p}=0.9275)$, after 4 weeks of treatment Malassezia colony formation was significantly reduced at scalp sides treated with verum shampoo compared to sides treated with placebo $\left(\mathrm{t}_{4}: \mathrm{p}=0.0016\right.$; Figure 3). In case the verum shampoo was used in combination with the tonic, formation of Malassezia colonies was significantly decreased at the treated sides not only after 4 but already after 2 weeks $\left(\mathrm{t}_{2}: \mathrm{p}=0.0151, \mathrm{t}_{4}: \mathrm{p}=\right.$ 0.0001).

Even though treatment with the verum shampoo/tonic combination in comparison to verum shampoo alone showed a reduced number of colonies after 4 weeks, this difference was not significant. After completion of the post-treatment phase, anti-fungal activity, which had been present during the treatment phase, could no longer be detected in any test group. 

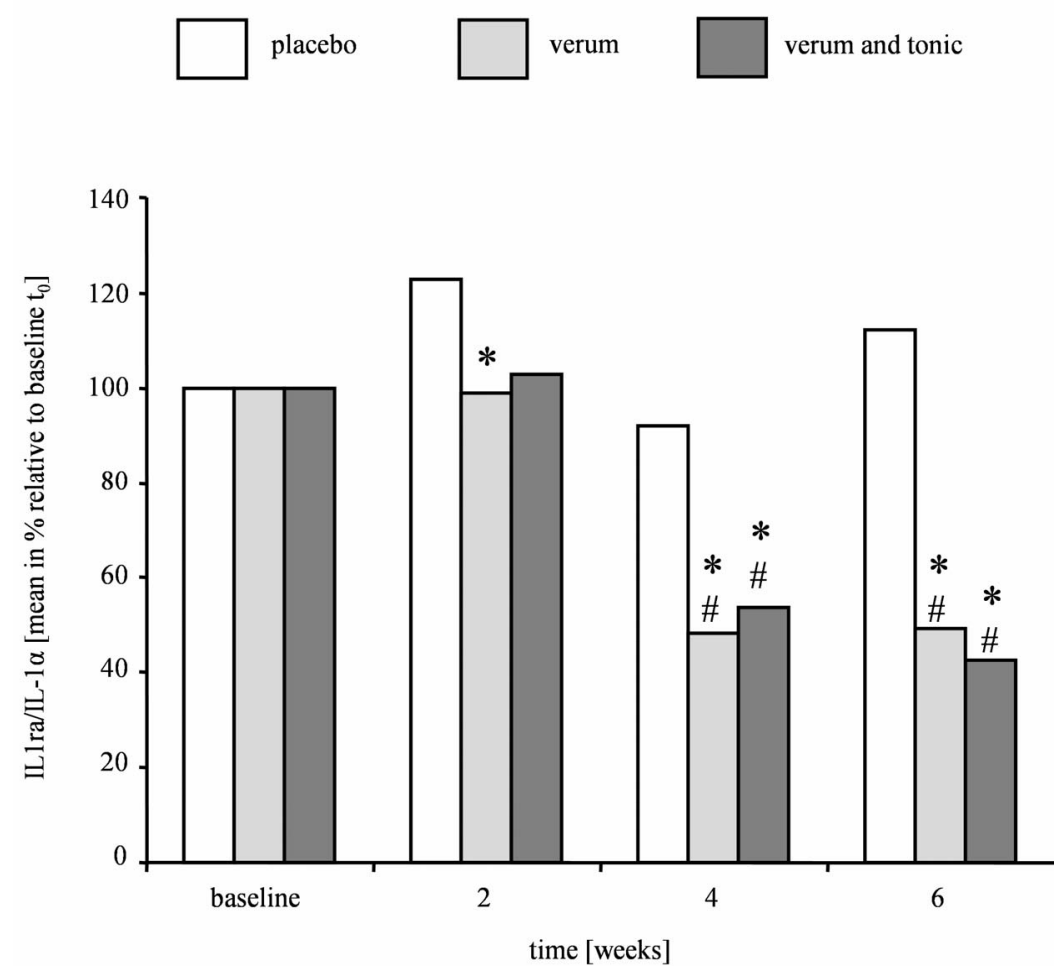

(a)
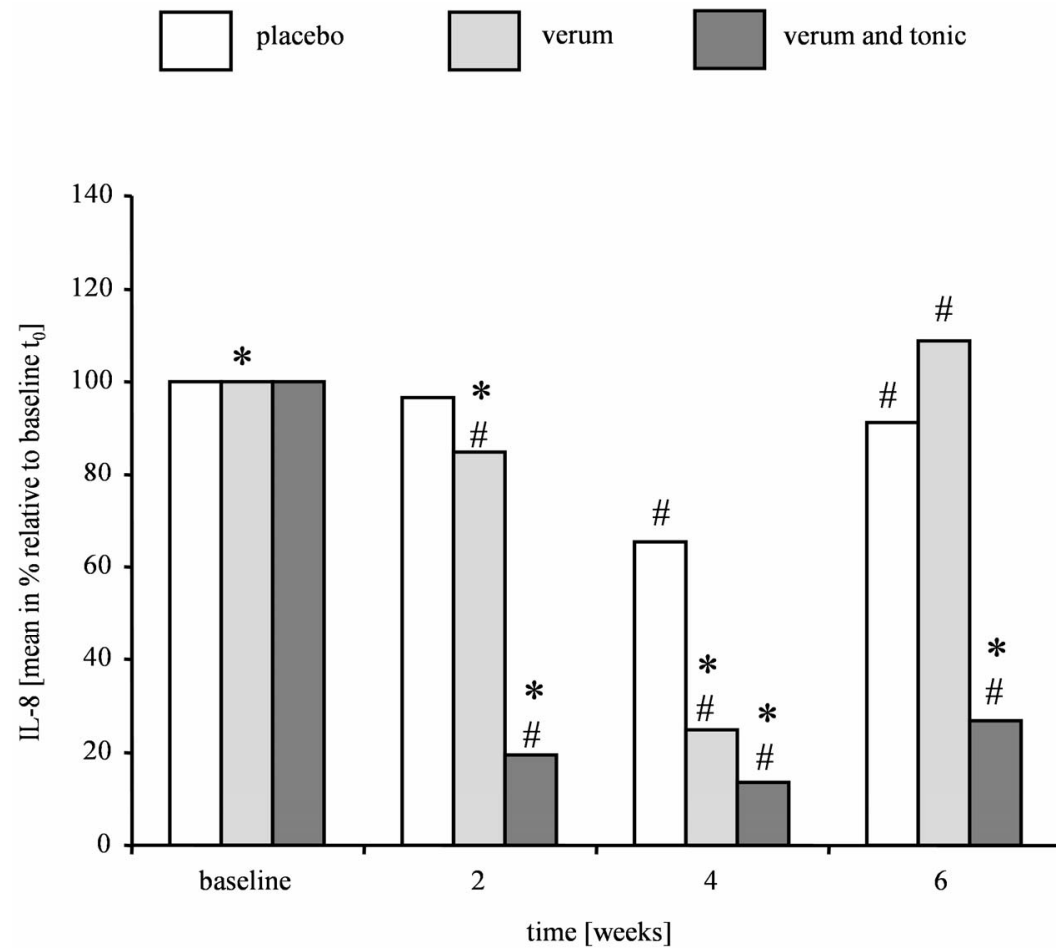

(b)

Figure 2. Levels of pro-inflammatory markers in volunteers with strong or very strong dandruff affection after treatment with placebo, verum, or verum shampoo in combination with tonic. (a) IL-1ra/IL-1 $\alpha$ levels; (b) IL-8 levels. Results are shown as mean in $\%$ relative to baseline $t_{0}(n=20$ (placebo shampoo), 19 (verum shampoo), 17 (verum shampoo and tonic)). Significant differences with respect to placebo shampoo are marked with an asterisk (* for $p \leq 0.05)$ and with respect to baseline $t_{0}$ with a hash ( ${ }^{\#}$ for $p \leq 0.05$ ). 

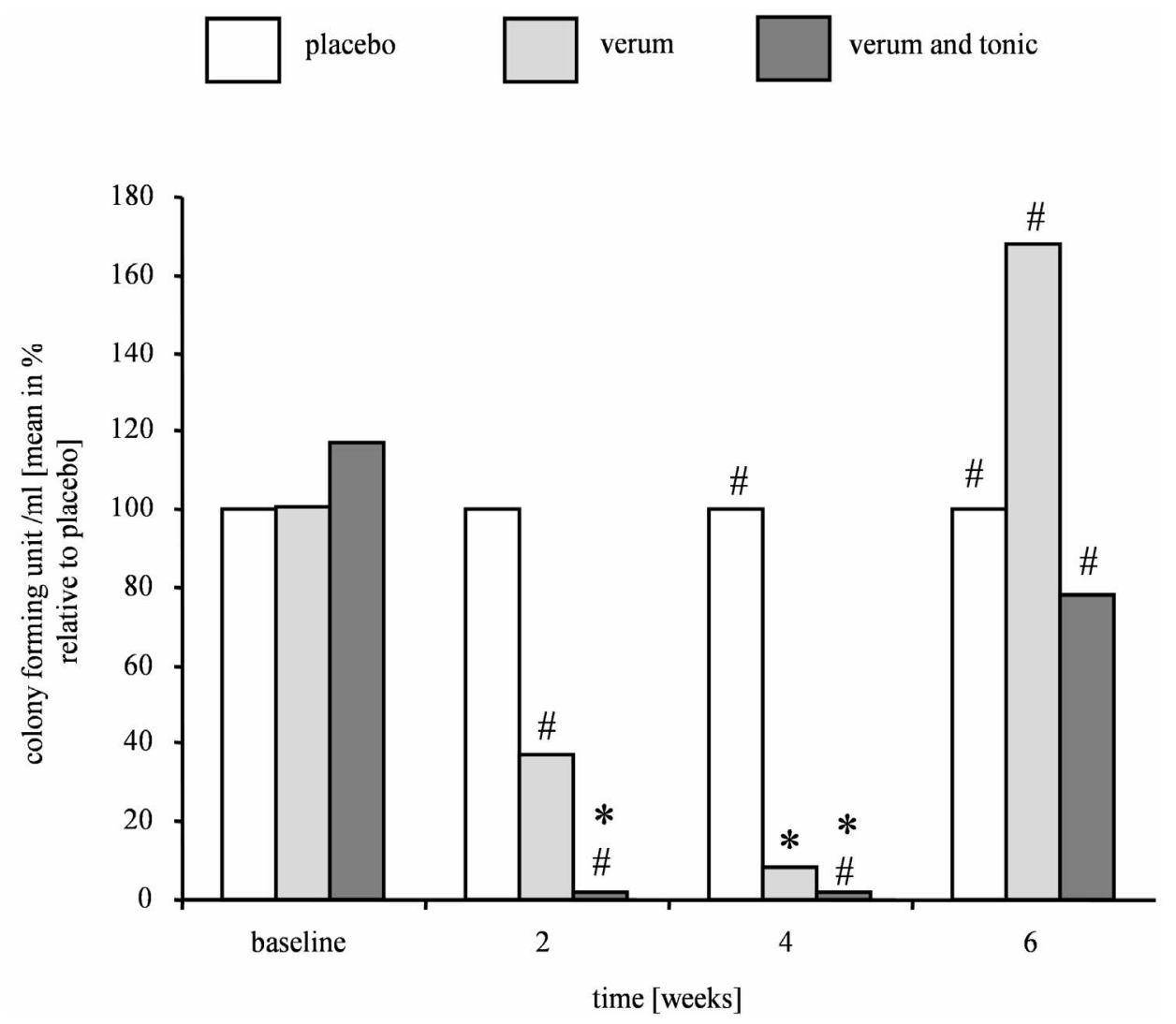

Figure 3. Anti-fungal activity of placebo, verum or verum shampoo in combination with tonic as detected in the Malassezia colony-forming assay. Results are shown as mean in \% relative to placebo treatment $(n=19-24)$. Significant differences with respect to placebo shampoo are marked with an asterisk (* for $p \leq 0.05)$ and with respect to baseline $t_{0}$ with a hash $\left({ }^{\#}\right.$ for $p \leq$ 0.05).

\section{Discussion}

The aim of an anti-dandruff treatment is to restore scalp health [14]. One requirement of an effective treatment is the removal of loosely attached scales from the scalp and subsequent growth inhibition of Malassezia species. However, with respect to anti-dandruff shampoos it is difficult to deliver actives directly to the human scalp due to the high hair density. Also, since the average resident time for shampoo on the scalp is only $30 \mathrm{~s}$ [15] and the application of a shampoo is always followed by a rinsing step, it is challenging to achieve a sufficient substantivity of actives. The amount of actives that remain on the scalp surface after rinsing is assumed to be crucial for longterm anti-dandruff effects [16]. A leave-on formulation increases the retention time of active ingredients compared to shampooing alone leading to an enhanced efficacy.

Therefore, a combination of shampooing and subsequent application of a leave-on tonic might provide an extra effective treatment option.

Several studies investigated the efficacy of anti-dandruff shampoos containing piroctone olamine or climbazole alone or in combination with other actives [17-19]. A shampoo comprising of $0.5 \%$ piroctone olamine and $0.45 \%$ climbazole showed an anti-fungal effectiveness comparable to shampoos containing particulate zinc pyrithione. After 4 weeks treatment, a sustained reduction of flaking and scalp itching was documented for this shampoo, as well as hair conditioning advantages [20]. Based on these results, we chose a shampoo containing piroctone olamine and climbazole for our in vivo study and also incorporated piroctone olamine into the tonic.

Standard dandruff formulations containing anti-mycotics only indirectly improve a disturbed stratum corneum (SC). A further treatment with actives, which restore SC condition, might optimize scalp health more effectively [14]. Also, a SC barrier dysfunction triggers the secretion of pro-inflammatory cytokines causing cutaneous (micro)-inflammation. To combat this inflammation, we added the potent anti-inflammatory active licochalcone A to the tonic. Licochalcone A, derived from Glycyrrhiza inflata root extract, has been shown to exert anti-inflammatory effects in vitro and in vivo [1012]. As shown in our laboratory, application of a leaveon tonic containing urea, lactate, polidocanol, and licochalcone A significantly improved scalp condition of dry, 
itchy, and subclinically inflamed scalp [13]. Since itchinduced scratching additionally exacerbates an impaired SC barrier function, the local anesthetic polidocanol [9] was incorporated into the tonic. A topical formulation containing the non-ionic surfactant polidocanol in combination with the humectant urea has already been successfully applied to relief pruritus [8].

In this study, we test the hypothesis that treatment with an anti-dandruff shampoo containing the actives piroctone olamine/climbazole and polidocanol in combination with a leave-on tonic containing the actives 1) piroctone olamine, 2) licochalcone $\mathrm{A}$, and 3) polidocanol exerts beneficial effects in subjects suffering from moderate to severe dandruff.

To address our hypothesis, we performed an in vivo split head design study and investigated the effect of scalp treatment with an anti-dandruff shampoo alone or the anti-dandruff shampoo combined with the antidandruff tonic against a placebo shampoo. After 4 weeks, expert grading confirmed that dandruff scores in volunteers treated with verum shampoo were significantly reduced compared to volunteers who had applied the placebo shampoo alone. This significant decrease in the amount of dandruff was demonstrated for the group who had applied the verum shampoo in combination with the tonic already after 2 weeks of treatment. After completion of the post-treatment phase, the significant dandruff reduction was better maintained by the rinse-off/ leave-on combination compared to verum shampoo. This fact suggests a more sustainable effect exerted by the combination.

Next, we investigated if these results were supported by a corresponding anti-fungal activity of test products. Using a Malassezia colony-forming assay for the investigation of scalp wash-ups, we showed that a 4-week treatment with verum compared to placebo shampoo yielded a highly reduced number of Malassezia colonies. In case the verum shampoo was used in combination with the tonic, formation of yeast colonies was significantly decreased at treated sites not only after 4 , but already after 2 weeks. These data correspond well with the results obtained from expert grading demonstrating a more rapid and better dandruff reduction exerted by the combination treatment. During the post-treatment phase, application of anti-fungal actives ceased. At $\mathrm{t}_{6}$, Malassezia colony forming units had returned to baseline or even exceeded baseline level. An anti-fungal activity, shown during treatment with the verum shampoo as well as the verum shampoo/tonic combination, could not be demonstrated any longer. These results were consistent with a slightly increasing dandruff score determined after the completion of the post-treatment phase indicating that the re-growth of Malassezia is correlated with dandruff formation.
Dandruff symptoms are determined by assessment of scalp skin condition. To study underlying inflammatoryrelated processes in more detail, we focused on the determination of well-known inflammatory markers. An increased IL-1ra/IL- $1 \alpha$ ratio is a widely recognized marker of skin inflammations of different origins [21], and high levels of IL-8 have been linked to active inflammatory processes [22]. In the dandruff scalp, the IL-1 ra/IL-1 $\alpha$ ratio was significantly elevated, and IL-8 levels were found to be significantly augmented [6] as well. Increased IL- 8 levels have been associated with dandruff and other inflammatory scalp diseases, such as seborrhoeic dermatitis and psoriasis [23]. The IL-1ra/IL-1 $\alpha$ ratio and the inflammatory marker IL-8 can be used as indicators for the determination of dandruff severity $[6,24]$.

To gain a deeper understanding of molecular events underlying the symptoms of dandruff, we investigated alterations in the IL-1ra/IL- $1 \alpha$ ratio and in IL- 8 levels after treatment with the test formulations. Our results showed a significant reduction of the IL-1ra/IL-1 $\alpha$ ratio and also a significant decrease in IL-8 levels compared to placebo after 4 weeks of treatment with the verum shampoo and the verum shampoo/tonic combination. Although the amount of dandruff increased slightly and the Malassezia colony formation significantly again in the post-treatment phase in treated sites, the important skin inflammatory marker IL-1ra/IL-1 $\alpha$ still maintained at significantly reduced levels. This result indicates a sustained anti-inflammatory effect of both anti-dandruff treatments. In contrast to the verum shampoo/tonic combination (containing licochalcone A as potent anti-inflammatory active), the verum shampoo alone diminished only one of the analyzed pro-inflammatory dandruff markers implicating a stronger and more sustainable effect of the combination on scalp inflammation.

In summary, the combined treatment with a shampoo containing piroctone olamine and climbazole and a new leave-on tonic containing piroctone olamine and licochalcone A reduced dandruff-associated scalp flaking and Malassezia growth more rapidly and effectively than shampooing alone. On the molecular level, significantly decreased levels of pro-inflammatory markers were determined during treatments with verum shampoo alone or verum shampoo/tonic combination. After the post-treatment phase, the verum shampoo/tonic combination group maintained this significant decrease better than the verum shampoo alone suggesting a superior effect for the combination in the reduction of scalp inflammation.

\section{Acknowledgements}

We kindly thank Dr. Janina Lorenz for the development of the IL-8 assay. In addition, the authors would like to thank Dr. Sabine Oberst for study monitoring, Dr. Tho- 
mas Hillemann and Bernhard Oltrogge for supporting the study organization. We further thank Institute Dr. Schrader Creachem GmbH, Holzminden, Germany for conducting the in vivo split head design anti-dandruff study.

\section{REFERENCES}

[1] J. R. Schwartz, C. M. Cardin and T. L. Dawson, "Dandruff and Seborrheic Dermatitis," In: R. Baran and H. I. Maibach, Eds, Textbook of Cosmetic Dermatology, Martin Dunitz Ltd., London, 2004, pp. 259-272.

[2] S. C. Chen, J. Yeung and M. Chren, "Scalpdex. A Quality-of-Life Instrument for Scalp Dermatitis," Archives of Dermatology, Vol. 138, No. 6, 2002, pp. 803-807. doi:10.1001/archderm.138.6.803

[3] L. Misery, V. Sibaud, M. Ambronati, G. Macy, S. Boussetta and C. Taieb, "Sensitive Scalp: Does This Condition Exist? An Epidemiological Study," Contact Dermatitis, Vol. 58, No. 4, 2008, pp. 234-238. doi:10.1111/j.1600-0536.2007.01288.x

[4] J. C. Szepietowski, A. Reich, E. Wesołowska-Szepietowska and E. Baran, "Quality of Life in Patients Suffering from Seborrheic Dermatitis: Influence of Age, Gender and Education Level," Mycoses, Vol. 52, No. 4, 2009, pp. 357-363. doi:10.1111/j.1439-0507.2008.01624.x

[5] B. I. Ro and T. L. Dawson, "The Role of Sebaceous Gland Activity and Scalp Microfloral Metabolism in the Etiology of Seborrheic Dermatitis and Dandruff," Journal of Investigative Dermatology Symposium, Vol. 10, No. 3, 2005, pp. 194-197.

[6] K. Kerr, T. Darcy, J. Henry, H. Mizoguchi, J. R. Schwartz, S. Morrall, et al., "Epidermal Changes Associated with Symptomatic Resolution of Dandruff: Biomarkers of Scalp Health," International Journal of Dermatology, Vol. 50, No. 1, 2011, pp. 102-113. doi:10.1111/j.1365-4632.2010.04629.x

[7] S. Seité, J. Paries, P. Reygagne, Z. Hamidou, C. Jouanique, G. Perez-Pala, et al., "A Lipohydroxyacid-Containing Shampoo Improves Scalp Condition and Quality of Life in Patients with Seborrheic Dermatitis and Lightto-moderate Scalp Psoriasis," Journal of Cosmetic Dermatology, Vol. 8, No. 2, 2009, pp. 108-113. doi:10.1111/j.1473-2165.2009.00431.x

[8] G. Freitag and T. Höppner, "Results of a Postmarketing Drug Monitoring Survey with a Polidocanol-Urea Preparation for Dry, Itching Skin," Current Medical Research and Opinion, Vol. 13, No. 9, 1997, pp. 529-537. doi:10.1185/03007999709113326

[9] C. S. Leopold and H. I. Maibach, "Effect of Cutaneously Applied Nonionic Surfactants and Local Anesthetic Bases on Thermal Sensations," Pharmazie, Vol. 59, No. 1, 2004, pp. 50-54.

[10] I. Furuhashi, S. Iwata, S. Shibata, T. Sato and H. Inoue, "Inhibition by Licochalcone A, a Novel Flavonoid Isolated from Liquorice Root, of IL-1beta-Induced PGE2 Production in Human Skin Fibroblasts," Journal of Pharmacy and Pharmacology, Vol. 57, No. 12, 2005, pp. 16611666. doi:10.1211/jpp.57.12.0017
[11] L. Kolbe, J. Immeyer, J. Batzer, U. Wensorra, K. tom Dieck, C. Mundt, et al., "Anti-inflammatory Efficacy of Licochalcone A: Correlation of Clinical Potency and in Vitro Effects," Archives of Dermatological Research, Vol. 298, No. 1, 2006, pp. 23-30. doi:10.1007/s00403-006-0654-4

[12] T. M. Weber, R. I. Ceilley, A. Buerger, L. Kolbe, N. S. Trookman, R. L. Rizer, et al., "Skin Tolerance, Efficacy, and Quality of Life of Patients with Red Facial Skin Using a Skin Care Regimen Containing Licochalcone A," Journal of Cosmetic Dermatology, Vol. 5, No. 3, 2006, pp. 227-232. doi:10.1111/j.1473-2165.2006.00261.x

[13] D. Schweiger, C. Baufeld, P. Drescher, B. Oltrogge, S. Höpfner, J.-P. Vietzke, et al., "Efficacy of a New Tonic Containing Urea, Lactate, Polidocanol and Glycyrrhiza Inflata Root Extract against Symptoms of a Dry, Itchy and Subclinically Inflamed Scalp," Skin Pharmacology and Physiology, 2013 (unpublished).

[14] G. A. Turner, M. Hoptroff and C. R. Harding, "Stratum Corneum Dysfunction in Dandruff," International Journal of Cosmetic Science, Vol. 34, No. 4, 2012, pp. 298306. doi:10.1111/j.1468-2494.2012.00723.x

[15] P. Mayser, P. Haze, C. Papavassilis, M. Pickel, K. Gruender and E. Guého, "Differentiation of Malassezia Species: Selectivity of Cremophor EL, Castor Oil and Ricinoleic Acid for M. Furfur," British Journal of Dermatology, Vol. 137, No. 2. 1997, pp. 208-213. doi:10.1046/j.1365-2133.1997.18071890.x

[16] J. R. Schwartz, R. Shah, H. Krigbaum, J. Sacha, A. Vogt and U. Blume-Peytavi, "New Insights on Dandruff/Seborrhoeic Dermatitis: The Role of the Scalp Follicular Infundibulum in Effective Treatment Strategies," British Journal of Dermatology, Vol. 165, Suppl. 2, 2011, pp. 18-23. doi:10.1111/j.1365-2133.2011.10573.x

[17] E. Futterer, "Evaluation of Efficacy of Anti-Dandruff Agents," Journal of Cosmetic Science, Vol. 32, No. 6, 1981, pp. 327-338.

[18] M. Loden, "Role of Topical Emollients and Moisturizers in the Treatment of Dry Skin Barrier Disorders," American Journal of Clinical Dermatology, Vol. 4, No. 11, 2003, pp. 771-788. doi:10.2165/00128071-200304110-00005

[19] W. Wigger-Alberti, K. Kluge and P. Elsner, "Clinical Effectiveness and Tolerance of Climbazole Containing Dandruff Shampoo in Patients with Seborrheic Scalp Eczema," Praxis, Vol. 90, No. 33, 2001, pp. 1346-1349.

[20] T. Schmidt-Rose, S. Braren, H. Fölster, T. Hillemann, B. Oltrogge, P. Philipp, et al., "Efficacy of a Piroctone Olamine/Climbazol Shampoo in Comparison with a Zinc Pyrithione Shampoo in Subjects with Moderate to Severe Dandruff," International Journal of Cosmetic Science, Vol. 33, No. 3, 2011, pp. 276-282. doi:10.1111/j.1468-2494.2010.00623.x

[21] T. Terui, T. Hirao, Y. Sato, T. Uesugi, M. Honda, M. Iguchi, et al., "An Increased Ratio of Interleukin-1 Receptor Antagonist to Interleukin-1Alpha in Inflammatory Skin Diseases," Experimental Dermatology, Vol. 7, No. 6, 1998, pp. 327-334. doi:10.1111/j.1600-0625.1998.tb00332.x 
[22] O. Arican, M. Aral, S. Sasmaz and P. Ciragil, "Serum Levels of TNF-Alpha, IFN-Gamma, IL-6, IL-8, IL-12, IL-17, and IL-18 in Patients with Active Psoriasis and Correlation with Disease Severity," Mediators of Inflammation, Vol. 5, No. 5, 2005, pp. 273-279. doi:10.1155/MI.2005.273

[23] M. A. Perkins, C. W. Cardin, M. A. Osterhues and M. K. Robinson," A Non-Invasive Tape Absorption Method for Recovery of Inflammatory Mediators to Differentiate Normal from Compromised Scalp Conditions," Skin Re- search and Technology, Vol. 8, No. 3, 2002, pp. 187-193. doi:10.1034/j.1600-0846.2002.20337.x

[24] K. J. Mills, P. Hu, J. Henry, M. Tamura, P. Tiesman and J. $\mathrm{Xu}$, "Dandruff/Seborrhoeic Dermatitis Is Characterized by an Inflammatory Genomic Signature and Possible Immune Dysfunction: Transcriptional Analysis of the Condition and Treatment Effects of Zinc Pyrithione," British Journal of Dermatology, Vol. 166, Suppl. 2, 2012, pp. 33-40. doi:10.1111/j.1365-2133.2012.10863.x 